\title{
The 2:1 librating-circulating planetary configuration produced by a hybrid scenario
}

\author{
Jianghui $\mathbf{J i}^{1}$, Zhao Sun ${ }^{1,2}$, Sheng $\mathbf{J i n}^{1,2}$ and Niu Zhang ${ }^{1,2}$ \\ ${ }^{1}$ Purple Mountain Observatory, Chinese Academy of Sciences, Nanjing, 210008, China; \\ email: jijh@pmo.ac.cn. \\ ${ }^{2}$ Graduate School of Chinese Academy of Sciences, Beijing 100049, China
}

\begin{abstract}
Different migration scenario of two giant planets may play a major role in forming the diverse resonant planetary configurations. The studies on the HD 128311 and HD 73526 systems show that two gas giants are captured in a 2:1 resonance but not in apsidal corotation, because one of the resonant argument circulates over the dynamical evolution. Herein we explore potential scenarios to produce the 2:1 librating-circulating resonance configuration. In the simulations, we find that both colliding or scattering events at early stage of dynamical evolution can induce the configurations trapped into resonance. In this sense, the librating-circulating resonance configuration is more likely to form by a hybrid mechanism of scattering and collision.
\end{abstract}

Keywords. celestial mechanics, planetary systems: formation

\section{Introduction}

At present, four resonant pairs of planets (GJ 876, HD 82943, HD 128311 and HD 73526) are believed to be trapped in 2: 1 mean motion resonance (Marcy et al. 2001; Mayor et al. 2004; Vogt et al. 2005; Tinney et al. 2006). Numerical explorations, as well as theoretical analysis, show that the $2: 1$ resonance planetary configuration could be quite diverse as a result of migration in a slightly eccentric disc (Lee 2004; Kley et al. 2005). For example, the HD 128311 and HD 73526 systems are stabilized by the 2:1 resonance, but with a librating-circulating resonance configuration, which may be formed either through fast migration or migration with initial eccentricities or via a dynamical scattering event (Tinney et al. 2006).

\section{Model and Numerical Setup}

In the model, we consider the HD 128311 system as the fundamental framework. Here, we change the eccentricities of two giants as our initial eccentricities in order to emulate a slightly earlier state of this system (Zhang et al. 2010). The other parameters for the giant planets are the same as in (Sándor \& Kley 2006). The modified orbital parameters are dynamically stable at least over $10^{6}$ yr under mutual gravitational interaction. We assume $2-4$ terrestrial planets distributed in the range $0.3-0.75$ AU to explore this evolution. The eccentricities of the terrestrial planets are randomly adopted between 0.1 and 0.3 , and the terrestrial bodies have a total mass of $10-15 M_{\oplus}$. In each simulation, the terrestrial planets are in coplanar orbits reference to the giant planets. The other angles of the mean anomaly $M$ and the longitudes of periapse $\varpi$ are randomly distributed between $0^{\circ}$ and $360^{\circ}$ for each orbit.

We set up 120 simulations for two groups and use the hybrid symplectic integrator (Chambers 1999) in MERCURY package to integrate all orbits. In these runs, we consider that the collision and merging occurs when the minimum distance between any of 

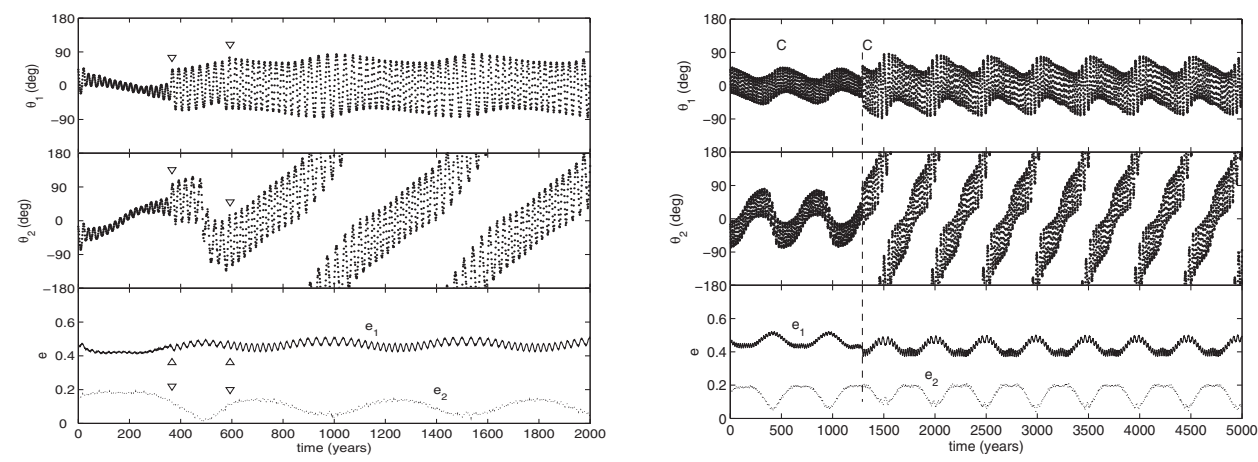

Figure 1. Time evolution for two simulations. Left panel: (1a) Evolution of the 2:1 mean motion resonance variables $\theta_{1}, \theta_{2}$, and the eccentricities of the two giants $e_{1}, e_{2}$, for Run 1 . The sign triangles label the time of two terrestrial objects that scattering happens. Right panel: (1b) The first upper letter ' $C$ ' denotes a merging event between T1 and T2. And the second collision occurs between T12 and the inner giant.

two objects is equal to or less than the summation of their physical radii. Most of the simulations are run for $10^{3}-10^{4} \mathrm{yr}$, but a few are extended to a longer integral time.

\section{Results}

Previous works on the HD 128311 and HD 73526 systems reported that the libratingcirculating resonance configuration are produced by scattering of an additional small planet through orbital migration of a sudden stop (Sándor \& Kley 2006; Sándor et al. 2007). We further show that collision and merger can play a major role in the evolution and both mechanisms can modify the resonance angles of the giants. The configuration engaged in the resonance is altered or destroyed. In the results, there are 16 simulations involved in producing librating-circulating resonance configuration, among which about $56 \%$ are shaped by mixed events of planet-planet scattering and merging (or collision). Details of the initial conditions for terrestrial planets may refer to Zhang et al. (2010).

We find that if the masses of the scattered terrestrial bodies are low, the changes of the resonance configurations may reinstate after some while. Generally, small planet scattering can increase the amplitude of the resonance angles. It is very likely that continuous scattering events of low-mass planets can alter the resonant configuration. Figure 1(a) shows the result of Run 1. Herein two terrestrial planets with equal masses of $m_{T 1}=m_{T 2}=5 M_{\oplus}$ first move about the giants. The $2: 1$ resonance of two giants is initially in symmetric configuration where $\left(\theta_{1}, \theta_{2}\right) \approx\left(0^{\circ}, 0^{\circ}\right)$. At the time the first terrestrial planet $\mathrm{T} 1$ is scattered at $366 \mathrm{yr}$, the amplitudes of the resonance angles become enlarged, but the apsidal corotation is maintained. Until the second terrestrial planet is scattered at $593 \mathrm{yr}$, soon after the first scattering event, the apsidal corotation is broken up. The eccentricities of two giants $e_{1}$ and $e_{2}$ fluctuate with large amplitudes, and our outcomes are well consistent with those of (Sándor \& Kley 2006; Sándor et al. 2007).

Figure 1(b) shows the evolution of Run 2. The first ' $C$ ' denotes a merging event between $\mathrm{T} 1$ and T2 at about $500 \mathrm{yr}$. The second collision occurs between T12 (as a merged larger body) and the inner giant at about $1300 \mathrm{yr}$. In this case, two resonant arguments $\theta_{1}$ and $\theta_{2}$ vary from a librating-librating phase into the librating-circulating state, and both of the eccentricities for two giants are slightly modulated. This shows that a terrestrial planet's colliding with a giant may finally shape librating-circulating configuration of mean motion resonances during the dynamical evolution. 


\section{Conclusions and Discussions}

In the late stage of planetary formation, scattering or colliding among planetesimals and embryos does frequently occur. If two giant planets are initially trapped in a 2:1 symmetric resonance and their eccentricities oscillate with large amplitudes, the collisions arising from the giants and other small bodies may change librating amplitudes of resonant angles over the evolution. If the apsidal corotation is destoyed, the configuration then turns into a librating-circulating state. Obviously, the greater mass of a perturbing terrestrial body may have more significant influence on the commensurable giant planets. In most runs, colliding and scattering events are found to increase or decrease the fluctuation in the amplitude of the resonant angles, even dramatically break up the whole system. In a word, the librating-circulating configuration of mean motion resonance is likely to generate by a hybid mechanism of colliding and scattering.

\section{Acknowledgements}

This work is financially supported by the National Natural Science Foundation of China (Grants 10973044, 10833001), the Natural Science Foundation of Jiangsu Province, and the Foundation of Minor Planets of Purple Mountain Observatory.

\section{References}

Chambers, J. E. 1999, MNRAS, 304, 793

Kley, W., Lee, M. H., Murray, N., \& Peale, S. J. 2005, A\&A, 437, 727

Lee, M. H. 2004, ApJ, 611, 517

Marcy, G. W., Butler, R. P., Fischer, D. A., et al. 2001, ApJ, 556, 296

Mayor, M., Udry, S., Naef, D., et al. 2004, A\&GA, 415, 391

Sándor, Z. \& Kley, W. 2006, A\&A, 451, L31

Sándor, Z., Kley, W., \& Klagyivik, P. 2007, A\& A, 472, 981, 28

Tinney, C. G., Butler, R. P., Marcy, G. W., et al. 2006, ApJ, 647, 594

Vogt, S. S., Butler, R. P., Marcy, G. W., et al. 2005, ApJ, 632, 638

Zhang, N., Ji, J. \& Sun, Z. 2010, MNRAS, 405, 2016 